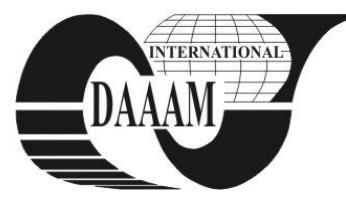

\title{
GRAPHICAL INTERPRETATIONS FUNCTIONS OF SEVERAL VARIABLES FOR USING IN THE TECHNOLOGICAL EXPERIMENTS
}

\author{
MACURA, D[usan]
}

Abstract: In the paper behaviou of a function present graphs of a functions by using mathematical analysis and graphical methods that include plotting points and determined symmetry. These techniques are adequate for obtaining a graph. We can use properties of the functions to predict some parameters of he technological experiments for example the height and speed of the manufacturing. The presentation functions are inspirative and motivated for the manufacturing experiments. We study important geometric properties of the curves

Key words: technological experiment, function, graph, behavior of a function

\section{INTRODUCTION}

We need to generalize the main ideas of calculus - limits, continuity, derivatives, and integrals - for technological experiments to relationships whose graphs may be curves or surfaces in three dimensional spaces. The relationships are expressed through functions whose domains or co-domains maybe multivariable (Macurova, 2009). We begin the study of graph functions in which the situation is reversed: eachelement of the domains a vector whereas are called realvalued functions of several variables. First, we examine how to graphically represent the function of two or three variables bythe use of level curves and level surfaces and continuity of partial differentiation.

\section{GRAPHICAL INTERPRETATION OF THE SOME FUNCTIONS POSSIBILITY OF EXPERIMENTAL TECHNOLOGICAL}

Let $f$ be a function of two variables. The graph of function $f$ is a set of all ordered triples $[x, y, f(x, y)]$, where ordered pairs are from domain of $f$.Consider the trace of the graph of $f$ on the plane $z=k$ as illustrated in Figure 1. If we project this trace onto the $x y$-plane, we obtain a curve $c$ with equation $f(x, y)=k$. We can see that if a point $[x, y, 0]$ moves along $c$, the corresponding function values $f(x, y)$ always equal $k$. We call $c$ a level curve of $f$.

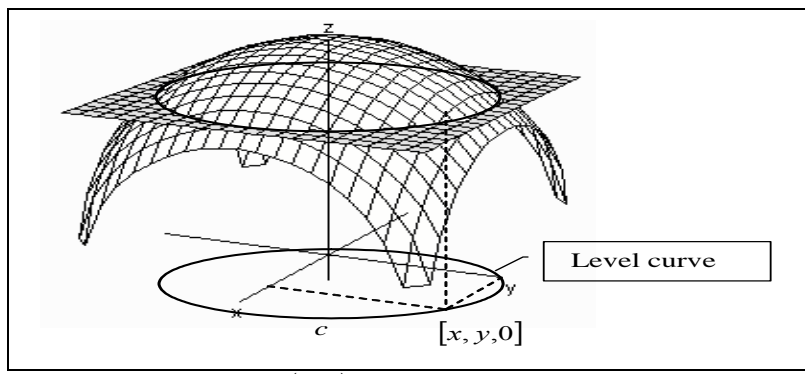

Fig. 1. Level curve $f(x, y)=k$
The presentation functions are inspirative for the dependencies of the experiments

Sketch the graph of $f(x, y)=y^{2}-x^{2}$ and sketch some level curve. The graph of $f$ is the hyperbolic paraboloid.

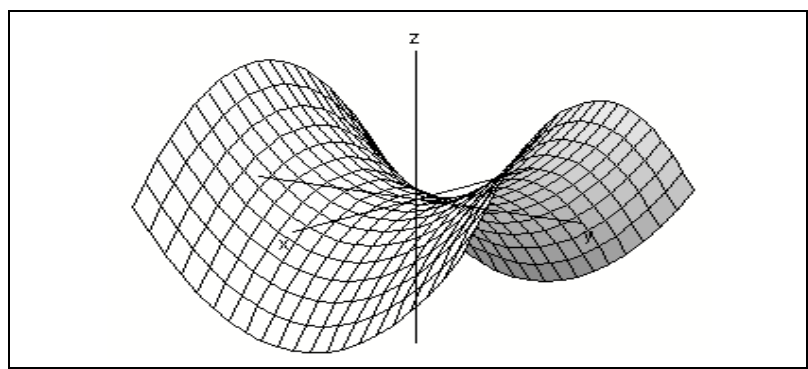

Fig. 2. Graph of function $f(x, y)=y^{2}-x^{2}$

The level curves in the $x y$-plane are the graphs of the equations $y^{2}-x^{2}=k$, for $k \in R$.

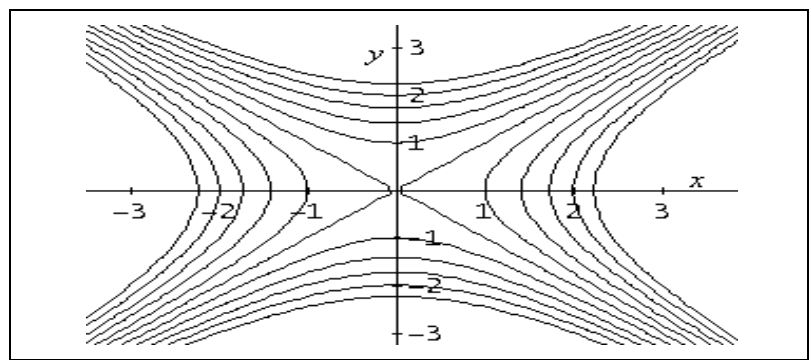

Fig. 3. Level curve $y^{2}-x^{2}=k$

We describe the domain of $f(x, y)=\sqrt{x^{2}+y^{2}-1}+\ln \left(4-x^{2}-y^{2}\right)$ and we sketch the graph of $f$. The domain $D$ is the set of all pairs $[x, y] \in E_{2}$ such that $x^{2}+y^{2}-1 \geq 0 \wedge 4-x^{2}-y^{2}>0$.

Thus the graph of $D$ is the set $D=\left\{[x, y] \in E_{2} ; x^{2}+y^{2} \geq 1 \wedge x^{2}+y^{2}<4\right\}$.

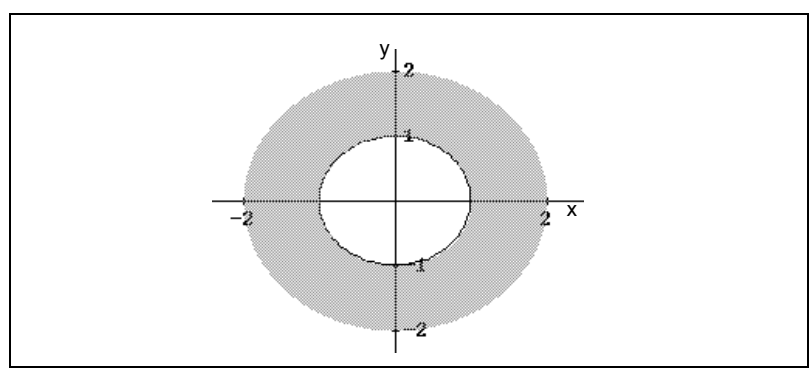

Fig. 4. Graph of domain $D$ of $f(x, y)=\sqrt{x^{2}+y^{2}-1}+\ln \left(4-x^{2}-y^{2}\right)$ 


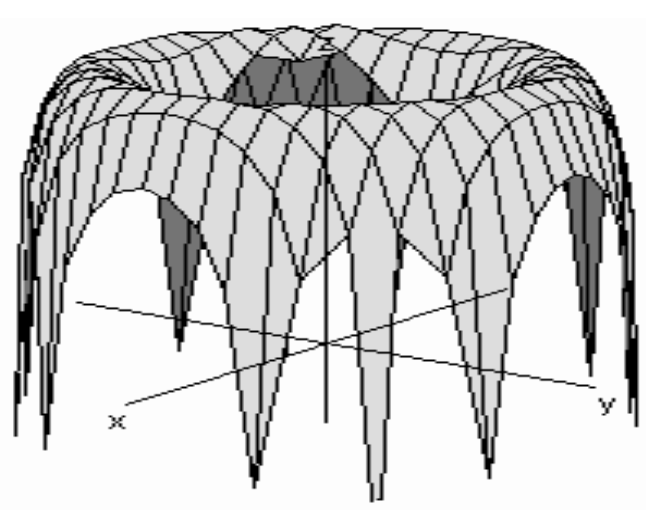

Fig. 5. Graph of function

$f(x, y)=\sqrt{x^{2}+y^{2}-1}+\ln \left(4-x^{2}-y^{2}\right)$

Consider the functions $f(x, y)=x^{y}, g_{1}(x, y)=x^{2}+y^{2}$, and $g_{2}(x, y)=x-y$. Express $f\left(g_{1}(x, y), g_{2}(x, y)\right)$ in terms of $x$ and $y$, and find the domain of the resulting composite function. Substituting $g_{1}(x, y)$ for $x, g_{2}(x, y)$ for $y$, in the expression for $f(x, y)$ then $f\left(g_{1}(x, y), g_{2}(x, y)\right)=\left(x^{2}+y^{2}\right)^{x-y}$, the domain is $E_{2}=R \times R-\{[0,0]\}$. The technological experiments are present by functions with two variables. The dependences can be approximated by formules of the functions in the present graphs. Technological process consists of a large set of hierarchic mutually linked subsystems acting a space and parameters by variables. It is often possible to describe their functioning analytically due to the complexity of a technological process.An important component of the analysis of the experiment is its decomposition into more parameters which can be independent variables. Nowadays, there is no formalized means of experiments decomposition. Which the decomposition of experiments the following set of techniques has been used in practice. Decomposition is carried out in various classifications. The same parameter can occur in various subexperiments (Wayene,1993). Mathematical model of the technological experiments is usually expressed by a formula present the finction with one reale variable.

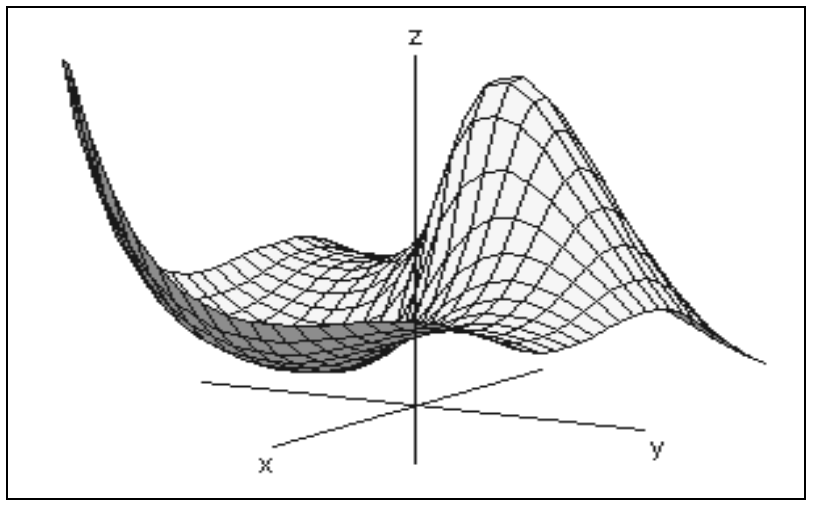

Fig. 6. Graph of $f(x, y)=\left(x^{2}+y^{2}\right)^{x-y}$

\section{CONCLUSION}

Computers can be programmed to show level curves and traces on various panes and to represent surface from various perspectives (Macurova, 2009). Several computers generated graphs are shown in Figure 1 to Figure 6. The graphs present in the paper are inspirative for experimental dependences in the technology experiment. For example the maximum deviation roughness of the cut surface $R z$ of the feed $f$ and of the cutting speed $v_{c}$ is the function $R z=R z\left(f, v_{c}\right)$ and the mathematical expression of the dependency is the mathematical model as function (Macurova, 2009). The explicit formule of the dependency of the maximum deviation roughness at the cut surface $R z$ at the cutting speed and the feed $f$ as mathematical formule not is in the literature. Technological experiments for th dependency of the durabilitz of the knife $T$ at the cutting speed $v_{c}$, at the feed $f$ is represented by mathematical models. The characterictics of the calculate parameters are approximating to characteristic of the linear model when the capacity are big sufficiency of the measuriny (Vasilko, 2004). The previous questions are the theme of the next investigation.

The characteristics of the calculate parameters are approximating to characteristic of the linear models henthe capacity are big sufficient than the non linear model is manipulate with the linear model (Macura, 2005). At present, a very topical problem area in many technological disciplines is that of process which are not amenable to sufficiently precise description

\section{REFERENCES}

Wayene, L. W. (1993). Operations Research, Wadsworth Publisching Company, ISBN 0-534-20971-8, California

Macurova, A. (2009). About solution of the non-linear differential equations, Technical University Kosice, ISBN, 978-80-8073-910-2, Kosice

Macurova, A. (2010). The roughness surface expressed by the mathematical model, Applied Surface Science, Vol. 356, no. 18 (2010), p. 5656 - 5658, ISSN 0169-4332

Vasilko, K. (2004). Technology of change measurement. Technical University of Kosice, ISBN 80-8073-160-8, Kosice

Macura, D. (2005). Function of Multivariable.University of Presov, ISBN 80-8068-321-2, Presov 